\title{
The Patched Intrinsic Tactile Object: a Tool to Investigate Human Grasps
}

\author{
Alessandro Serio ${ }^{1}$, Emanuele Riccomini ${ }^{1}$,Vincenzo Tartaglia ${ }^{1}$, Ioannis Sarakoglou ${ }^{2}$, \\ Marco Gabiccini ${ }^{3}$, Nikos Tsagarakis ${ }^{2}$ and Antonio Bicchi ${ }^{4}$
}

\begin{abstract}
In this paper we report on the development of a modular multi-DoF $F / T$ sensor and its use in the implementation of a sensorized object capable of multi-touch detection. The sensor is composed of six 6 -axis $\mathrm{F} / \mathrm{T}$ sensors spatially organized on the faces of a cube. Different calibration methods are presented to directly tackle the coupling phenomena inherent to the spatial organization of the faces and the lightweight construction of the sensor which would have, otherwise, degraded its accuracy. To assess the performances of the calibration methods, a comparison is reported with respect to the measurements obtained with a commercial force/torque sensor considered as ground truth (ATI Delta). Thanks to the modular design and the possibility to cover the sensitive faces with surface patches of different geometry, a variety of sensorized objects with different shapes can be realized. The peculiar feature that all the components of the contact wrench can be measured on each face with high accuracy, renders it a unique tool in the study of grasp force distribution in humans, with envisioned use both in neuroscience investigations and robotic applications.
\end{abstract}

\section{INTRODUCTION}

Human grasp investigation plays a paramount role both in neuroscience and robotics.

In neuroscience, human grasp characterization is useful to understand how our brain controls and adapts hand motions and forces applied to accomplish different types of task (see e.g., [1] and [2]).

In robotics, the knowledge and the studies of the human hand can inspire robot hand control algorithms, giving rise, very often, to bio-aware algorithms for controlling and employing these mechanical devices in different scenarios [3].

If, on the one hand, hand posture recordings are today pretty easy to obtain, e.g. with motion capture systems [4], [5], the measurements of forces and torques that the hand exerts on the object still remains a challenge. This is mainly due to the lack of devices to perform measurements of all components of the applied wrenches during a grasp.

1 A. Serio, E. Riccomini and V. Tartaglia are with Centro di Ricerca "E. Piaggio", Università di Pisa, 1 Largo L. Lazzarino, 56100 Pisa, Italy. \{e.riccominis, vi.tartaglia\} at gmail.com, a.serio at centropiaggio.unipi.it

${ }^{2}$ I. Sarakoglou and N. Tsagarakis are with the Department of Advanced Robotics (ADVR), Istituto Italiano di Tecnologia, Genova, Italy \{ioannis.sarakoglou, nikos.tsagarakis\} at it. it

${ }^{3}$ M. Gabiccini is with the Centro di Ricerca "E. Piaggio", Università di Pisa, 1 Largo L. Lazzarino, 56100 Pisa, Department of Civil and Industrial Engineering (DICI), Largo Lucio Lazzarino 1, Pisa and Departement of Advanced Robotics (ADVR), Italian Institute of Technology, 30 Via Morego, 16163 Genova, Italy. m.gabiccini at ing.unipi.it

${ }^{4} \mathrm{~A}$. Bicchi is with the Centro di Ricerca "E. Piaggio", Università di Pisa, 1 Largo L. Lazzarino, 56100 Pisa and Department of Advanced Robotics (ADVR), Italian Institute of Technology, 30 Via Morego, 16163 Genova, Italy. bicchi at centropiaggio.unipi.it

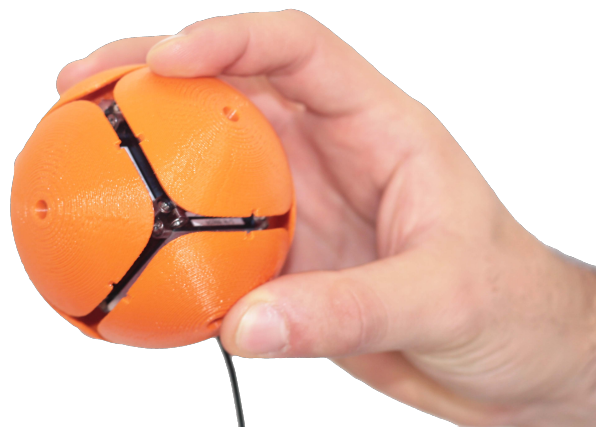

Fig. 1. The Patched Intrinsic Tactile Object with spherical shape, grasped by a human hand.

The existing devices for the force/torque characterization can be divided into two main groups.

In first group, we can collect the sensorized gloves capable to measure forces and torques from the "human hand side", as in [6]. Very often, these devices are composed by a stretchable tissue where deformation sensors are placed. Mostly, gloves are affected by the sliding between the human skin and the internal surface of the glove and are not capable of measuring more than one component of the wrench. Moreover, even in the most ergonomic devices, there is always a slight interference on the hand motions due the glove layer itself as well as to the sensors and associated electronics and wiring which may further constrain the movement of the hand.

In the other group, we can include the devices that allow to measure contact forces and/or torques from the "grasped object side". In this group, we can find sensorized objects consisting of a rigid structure where tactile sensor arrays, either piezoelectrical [7], [8] or capacitive [9], [10] are placed. These devices have a well-defined shape, and are thus capable to characterize human grasps performed on only one shape. Devices in this group can measure only a limited number of components of the contact wrench at each contact location.

In order to overcome these limitations, a new 36-axis multi-DoF F/T sensor has been designed at the Italian Institute of Technology that can be configured to cover its force sensing faces with surfaces of different shapes and sizes (for more details see [11], [12] and [13]).

The aim of the present work is to address the problem of calibrating the multi-DoF F/T sensor, reported in Fig. 1, evaluate its performances, and present results from trials of multi-fingered human grasps. The multi-DoF F/T sensor is 
(a) F/T Core
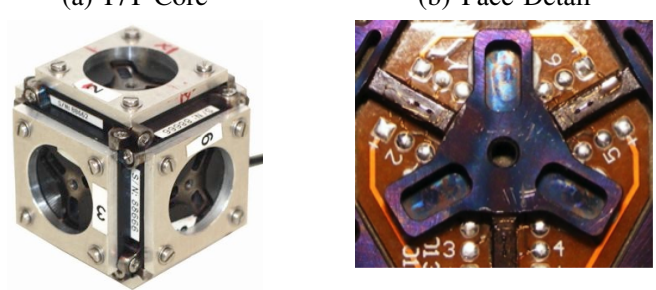

(c) Cube

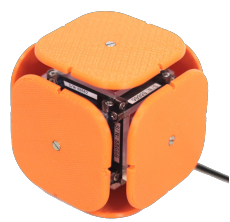

(d) Sphere

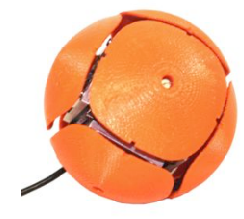

(e) Ellipsoid

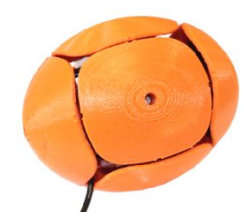

Fig. 2. In Fig. (a) we report the 36 axis F/T sensor which can be considered as the measure core of the patched intrinsic tactile object object. In Fig. (b) it is possible to notice a core face while in Figs. (c), (d) and (e) we show three different shape arrangement: a cube, a sphere and an ellipsoid, respectively.

a custom made 36-axis F/T sensor of cubical shape where each face is a 6-axis F/T strain gauge sensor. This device allows to measure all force and torque components during a grasp. Different patches can be attached on the sensor active faces (see Fig. 2) in order to obtain a multi-shaped sensorized object for characterizing grasps on different geometric surface primitives. The main limitation of the current layout is the fact that true contact measurements can only be recorded if one contact point (i.e., one finger) per patch is present: the envisioned solution relies, therefore, in the extreme miniaturization of the faces to obtain an intrinsic tactile sensitive skin.

In this multi-DoF F/T sensor, due to the spatial organization of the faces and the lightweight design, face crosscoupling phenomena occur. For this reason, conventional calibration methods - each 6-axis $\mathrm{F} / \mathrm{T}$ sensor is calibrated separately - are inapplicable, and therefore, two different calibration methods that explicitly account for coupling effects are presented.

The mathematical description of the various calibration methods is reported in Sec. III. Here, the ATI Delta commercial F/T sensor is employed to provide ground truth measurements. A metric of the error is introduced to compare the performances of the two different calibration methods. The results are discussed in Sec. IV. To identify the contact centroid on a surface patch of the corresponding face through the measurements of forces and torques, the algorithm based on [14] and [15] has been implemented. The contact point estimation results and some grasp force measurements during bimanual object manipulation are reported in Sec. VI. A video of the experiments is reported in the attachment: PITOExperiments.mp4.

\section{WORKING PRINCIPLE}

The core of the patched intrinsic tactile object consists of 6 force/torque sensors assembled in a cubical structure (see Fig. 2(a)). On each of the 6 sides of the core is

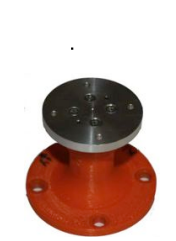

(a)
Custom Sensor Side

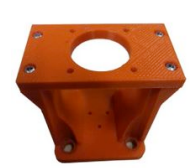

(b)

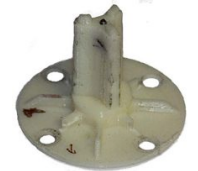

(c)
ATI Sensor Side

Fig. 3. Flange Type 1 (a), flange Type 2 (b) and flange Type 3 (c).

mounted an interaction face (Fig. 2(b)). By mounting different contact patches, simple geometric primitives such as a cube (Fig. 2(c)), a sphere (Fig. 2(d)) and an ellipsoid (Fig. 2(e)) or more complex shapes with arbitrary surface geometries can be created. This facilitates experimentation with several object shapes without the need for a dedicated measurement instrument or extensive apparatus changes for each desired geometry. The instrumented core contains all the sensors, instrumentation, conditioning electronics and communication interface for direct connection to a computer. The 6 force/torque sensing elements of the core measure the applied loads by monitoring the mechanical deflection of the measuring structure. The heart of the measuring device is a 3 spokes on a hub arrangement spaced at $120^{\circ}$, where are mounted 36 semiconductor strain gauges in 6 pairs (half Wheatstone bridge configuration). For more details see also figs. 1, 2 and 3 of [12].

\section{CALIBRATION METHODS}

Assuming a linear map between the space of counts (strain gauge measurements) and the space of the wrenches applied to the mechanical structure where the strain gauges are mounted, the calibration procedure accounts for identifying this linear map in the form of a calibration matrix. This assumption was proved to be reasonable by the tests performed in Sec. IV. The calibration is performed using an ATI Delta force/torque sensor for measuring ground truth wrenches applied on the custom sensor.

With reference to Fig. 4, the calibration structure is composed of a fixed frame, where the ATI Delta sensor is attached, and a flange that connects the custom sensor to the ATI Delta. The custom sensor can be calibrated in the reference system of the ATI Delta sensor $\left\{S_{A}\right\}$ or in the local reference system of each face $\left\{S_{j}\right\}$, with $j=1, \ldots, 6$.

The first flange (Fig. 3-(a)) is fixed to the custom sensor frame and does not allow to apply a wrench on the connected face. The second flange (Fig. 3-(b)) is fixed on the custom sensor frame but allows, through a hole, to apply a wrench on the corresponding connected face. The third flange (Fig. 3 -(c)) is fixed to one face of the custom sensor and allows to apply a wrench to all the faces except the connected one. This, however, is persistently excited when a wrench is applied to any other face due to the load path through the sensor towards the fixed frame.

Due to the coupling between the wrench applied to one face and gauge readings on the others, we directly tackled the 


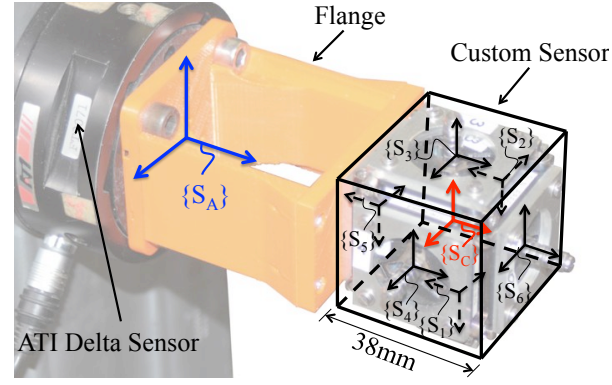

Fig. 4. Calibration structure with ATI Delta and custom made sensor reference systems.

problem of calibrating the whole sensor at once. Two methods were tested, as described in Sec. III-A and III-B, mainly to discern if different load paths through the sensor structure could have consequences on the calibration accuracy: these effects could not be easily estimated beforehand.

\section{A. Loaded Frame Calibration}

In this calibration method we employ the flange reported in Fig. 3-(b). The flange can be connect to any face. A wrench is applied on each face at a time in sequence, starting from active face 1 to active face 6 . In this way, a single calibration matrix is obtained for the whole device. Supposing to collect load wrenches $W_{i}^{S_{A}}$ (with $i=1, \ldots, 6$ ) applied to each face, the structure of the obtained wrench matrix is

$$
\hat{W}=\left[\begin{array}{c|c|c|c|c|c}
W_{1}^{S_{A}} & 0 & 0 & 0 & 0 & 0 \\
0 & W_{2}^{S_{A}} & 0 & 0 & 0 & 0 \\
0 & 0 & W_{3}^{S_{A}} & 0 & 0 & 0 \\
0 & 0 & 0 & W_{4}^{S_{A}} & 0 & 0 \\
0 & 0 & 0 & 0 & W_{5}^{S_{A}} & 0 \\
0 & 0 & 0 & 0 & 0 & W_{6}^{S_{A}}
\end{array}\right],
$$

where $0 \in \mathbb{R}^{6 \times 1}$ is a vector of zero elements and $W_{i}^{S_{A}} \in \mathbb{R}^{6 \times 1}$ is the wrench vector applied on the $i$-th face and expressed in the reference system $\left\{S_{A}\right\}$.

Consequently, we collect the strain gauge measurements in a matrix

$$
\hat{S}=\left[\begin{array}{c|c|c|c|c|c}
S_{1}^{1} & S_{1}^{2} & S_{1}^{3} & S_{1}^{4} & S_{1}^{5} & S_{1}^{6} \\
S_{2}^{1} & S_{2}^{2} & S_{2}^{3} & S_{2}^{4} & S_{2}^{5} & S_{2}^{6} \\
S_{3}^{1} & S_{3}^{2} & S_{3}^{3} & S_{3}^{4} & S_{3}^{5} & S_{3}^{6} \\
S_{4}^{1} & S_{4}^{2} & S_{4}^{3} & S_{4}^{4} & S_{4}^{5} & S_{4}^{6} \\
S_{5}^{1} & S_{5}^{2} & S_{5}^{3} & S_{5}^{4} & S_{5}^{5} & S_{5}^{6} \\
S_{6}^{1} & S_{6}^{2} & S_{6}^{3} & S_{6}^{4} & S_{6}^{5} & S_{6}^{6}
\end{array}\right],
$$

where $S_{i}^{j} \in \mathbb{R}^{6 \times 1}$, with $i=1, \ldots, 6$ and $j=1, \ldots, 6$ is the strain gauge measurements on $i$-th face applying the $j$-th wrench. Matrices (1) and (2) are related by

$$
\hat{W}=\hat{C} \hat{S}
$$

were $\hat{C} \in \mathbb{R}^{36 \times 36}$ is the calibration matrix. From (3), it is possible to compute the calibration matrix $\hat{C}$ as

$$
\hat{C}=\hat{W} \hat{S}^{\dagger}
$$

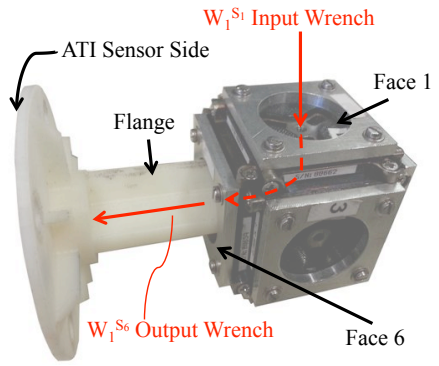

Fig. 5. Loaded diagram for Unloaded Frame Calibration.

where $\hat{S}^{\dagger}$ is the pseudo-inverse of $\hat{S}$.

With this assumption equation (4) can be rewritten as

$$
\hat{C}=\hat{W}_{k} \hat{S}_{k}^{\dagger}
$$

where $\hat{W}_{k} \in \mathbb{R}^{(36 \cdot 6) \times k}$ and $\hat{S}_{k} \in \mathbb{R}^{(36 \cdot 6) \times k}$ are the wrench and the strain gauge measurements matrix, respectively. The calibration matrix $\hat{C} \in \mathbb{R}^{36 \times 36}$ and $k$ is the number of the samples, i.e the number of the wrenches applied.

In order to ensure a proper exploration of the wrench space (with positive effects on the calibration matrix condition number) a large amount of samples $\left(k \sim 10^{4}\right)$ were collected. Since we were not able to exactly replicate the same loading samples in the different tests, we relied on applying a comparable set of perturbations in the same range. This range was set choosing maximum force norm values in the order of the full scale for the envisioned grasp analysis application. Details on this are provided in Sec IV.

\section{B. Unloaded Frame Calibration}

In this calibration method, the active face connected to the ATI Delta cannot be directly excited but it is affected by the wrenches applied on the other active faces. The flange reported in Fig. 3-(c) is used in this case. To make things simple, let us suppose to calibrate the custom sensor fixing the face 6 to the ATI Delta: the loaded diagram is reported in Fig. 5. The wrenches applied are measured by ATI Delta and are consequently expressed in the reference system $\left\{S_{A}\right\}$ $\left(W_{i}^{S_{A}}\right.$, with $\left.i=1, \ldots, 5\right)$. Upon applying an input wrench on face 1, an output wrench on face 6 is recorded (see Fig. 5); thanks to the equilibrium of a rigid body we get

$$
W_{1}^{S_{A}}=-W_{6}^{S_{A}}
$$

The calibration method is performed in the local reference system of each active face. Consequently, the wrenches applied in the local reference systems are computed with the adjoint transformations [16][17] as

$$
W_{1}^{S_{1}}=A d_{g_{S_{A} S_{1}}}^{T} W_{1}^{S_{A}} \quad W_{1}^{S_{6}}=A d_{g_{S_{A} S_{6}}^{T}}^{T} W_{1}^{S_{A}},
$$

where $W_{1}^{S_{1}}$ and $W_{1}^{S_{6}}$ are the wrenches applied on face 1 in reference system $\left\{S_{1}\right\}$ and $\left\{S_{6}\right\}$, respectively. The same procedure is performed for each face. Thus, collecting the wrenches applied $W_{i}^{S_{j}}$ on the active face $i$ (with $i=1, \ldots, 5$ ) and expressed in reference system $\left\{S_{j}\right\}$ with $j=1, \ldots, 6$ a wrench matrix is obtained as 


$$
\tilde{W}=\left[\begin{array}{c|c|c|c|c}
W_{1}^{S_{1}} & 0 & 0 & 0 & 0 \\
0 & W_{2}^{S_{2}} & 0 & 0 & 0 \\
0 & 0 & W_{3}^{S_{3}} & 0 & 0 \\
0 & 0 & 0 & W_{4}^{S_{4}} & 0 \\
0 & 0 & 0 & 0 & W_{5}^{S_{5}} \\
-W_{1}^{S_{6}} & -W_{2}^{S_{6}} & -W_{3}^{S_{6}} & -W_{4}^{S_{6}} & -W_{5}^{S_{6}}
\end{array}\right],
$$

where $0 \in \mathbb{R}^{6 \times 1}$ is a vector of zero elements.

Consequently the strain gauge measurements matrix is

$$
\tilde{S}=\left[\begin{array}{c|c|c|c|c}
S_{1}^{1} & S_{1}^{2} & S_{1}^{3} & S_{1}^{4} & S_{1}^{5} \\
S_{2}^{1} & S_{2}^{2} & S_{2}^{3} & S_{2}^{4} & S_{2}^{5} \\
S_{3}^{1} & S_{3}^{2} & S_{3}^{3} & S_{3}^{4} & S_{3}^{5} \\
S_{4}^{1} & S_{4}^{2} & S_{4}^{3} & S_{4}^{4} & S_{4}^{5} \\
S_{5}^{1} & S_{5}^{2} & S_{5}^{3} & S_{5}^{4} & S_{5}^{5} \\
S_{6}^{1} & S_{6}^{2} & S_{6}^{3} & S_{6}^{4} & S_{6}^{5}
\end{array}\right],
$$

where $S_{i}^{j} \in \mathbb{R}^{6 \times 1}$, with $i=1, \ldots, 6$ and $j=1, \ldots, 5$ are the strain gauge measurements on $i$-th face applying the $j$-th wrench. Matrices (8) and (9) are related by

$$
\tilde{W}=\tilde{C} \tilde{S},
$$

where $\tilde{C} \in \mathbb{R}^{36 \times 36}$ is the calibration matrix. $\tilde{C}$ as

From (10), it is possible to compute the calibration matrix

$$
\tilde{C}=\tilde{W} \tilde{S}^{\dagger},
$$

where $\tilde{S}^{\dagger}$ is the pseudo-inverse of $\tilde{S}$.

With this assumption Eq.(11) can be rewritten as

$$
\tilde{C}=\tilde{W}_{k} \tilde{S}_{k}^{\dagger},
$$

where $\tilde{W}_{k} \in \mathbb{R}^{(36 \cdot 6) \times k}$ and $\tilde{S}_{k} \in \mathbb{R}^{(36 \cdot 6) \times k}$ are the wrench and the strain gauge measurements matrix, respectively. The calibration matrix $\tilde{C} \in \mathbb{R}^{36 \times 36}$ and $k$ is the number of the samples, i.e the number of the wrenches applied.

As before, in order to ensure a proper exploration of the wrench space (with positive effects on the calibration matrix condition number) a large amount of samples $\left(k \sim 10^{4}\right)$ were collected.

\section{CALIBRATION RESULTS}

In this section we present the results for the Loaded Frame and Unloaded Frame Calibration methods III-A, III-B. Tests were performed (i) to assess the accuracy in discriminating the faces where the wrench is applied, and (ii) to test the precision of the measurements w.r.t the ATI Delta sensor. The experiments were performed: (i) loading only one face at a time, to check decoupling of the faces, and to identify the touched face; (ii) loading at least two faces simultaneously to simulate the squeezing effect caused by human grasp (squeeze experiment). The forces applied are in a range of $\pm 15 \mathrm{~N}$ while the torques are in a range of $\pm 0.1 \mathrm{Nm}$ (i.e in the range of human grasp).

For space limitations, only the results of some experiments are reported. The index employed for the estimation of the overall performances is, again for brevity, only the Root Mean Square Error (RMSE) of both forces and torques. The complete set of results are available from the authors upon request.

\section{A. Loaded Frame Calibration results}

As evident from Fig. 6, the Loaded Frame Calibration allows to identify the touched face by force readings. For each face, of the custom made sensor a number $k=100$ of samples was recorded. The $i$-th window, with $i=1, \ldots, 6$, relates the touched face to the samples. These windows also indicate the decoupling of the faces. The comparison plots in Fig. 7 and Fig. 9 also indicate the good performance of custom made sensor compared with the ATI Delta. Fig. 8 and Fig. 10 show that there is no notable influence of the choice of the face attached to the flange Type 2 in terms output accuracy quality (see Fig. 3-(b)). In Fig. 11 and Fig. 12 the RMSE index for the squeeze experiment are reported. From this, it can also be deduced that the performance of the custom made sensor remains approximately unchanged when two faces are loaded simultaneously.
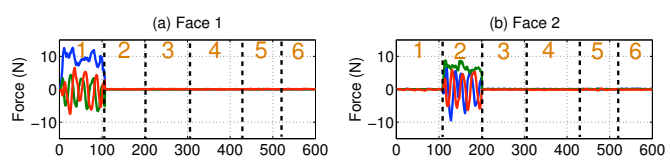

$$
\text { (c) Face } 3
$$
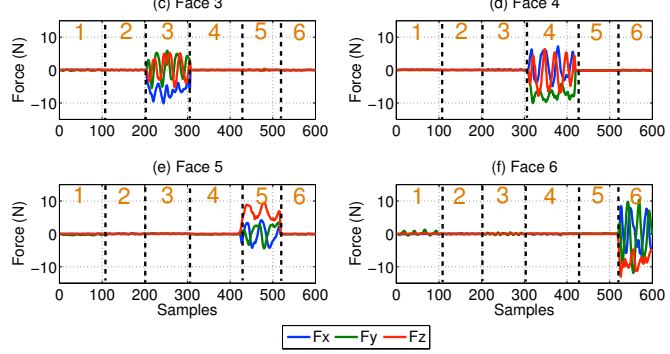

Fig. 6. Loaded Frame Calibration III-A - Forces - Identification of the loaded face - Only one face at a time is loaded.

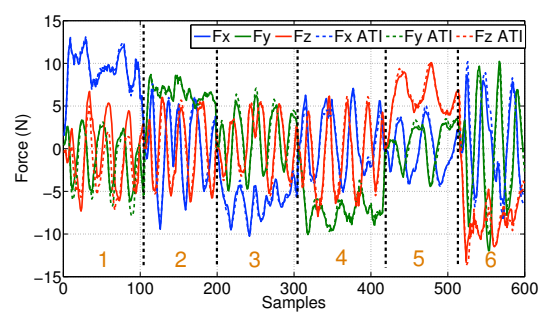

Fig. 7. Loaded Frame Calibration III-A - Forces - Comparison - Only one face at a time is loaded.

\section{B. Unloaded Frame Calibration results}

In Fig. 13 and Fig. 14 the RMSE index is depicted when a single face is loaded, upon changing the face connected to Type 3 flange (see Fig. 3-(c)). These experiments show that the performances of the custom made sensor with Unloaded Frame Calibration are superior to that of the Loaded Frame Calibration because, approximately, the RMSE for the force 


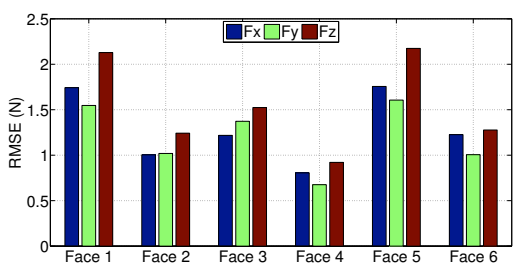

Fig. 8. Loaded Frame Calibration III-A - Forces - RMSE - Only one face at a time is loaded.

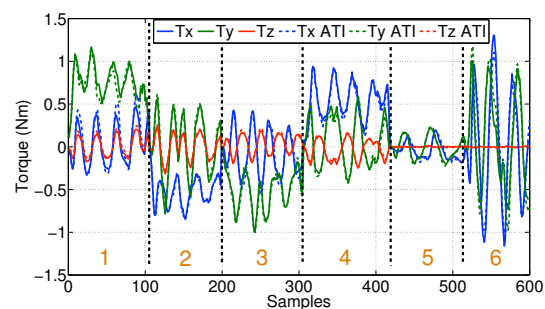

Fig. 9. Loaded Frame Calibration III-A - Torques - Comparison - Only one face at a time is loaded.

measurements is lower than $1 \mathrm{~N}$ and RMSE of moment measurements is lower than $0.17 \mathrm{Nm}$. It can be argued that this is due to the more favourable routes taken by the lines of force in this case, mainly linked to the limited involvement of the screw connections of the outer frame, which appears to be a source of nonlinearity and hysteresis.

Fig. 17 allows to identify the touched faces in the squeeze experiment by the torques, when the face 6 is connected to the flange Type 3 (see Fig. 3-(c)). The $i$-th window, with $i=1, \ldots, 8$, shows the decoupling of the faces and the capability of the custom made sensor to identify the faces loaded simultaneously (faces 1-3, faces 2-4, faces 3 5 , faces $4-5$, faces $2-5$, faces $1-5$, faces $1-4$ and faces $1-$ 2). Fig. 15 and Fig. 18 show the comparison between force and torque measurements of the custom made sensor and those of the ATI Delta sensor, in the squeeze experiment. In this case, the performances of the custom made sensor in the squeeze experiment, as shown in Fig. 16 and Fig. 19, are satisfactory and quite similar to the Loaded Frame Calibration performance. For all the experiments (see Fig. 13, Fig. 14, Fig. 16 and Fig. 19) the performance of the Unloaded Frame Calibration with face 1 connected to the flange is worse than the other faces. A possible explanation is the presence of the electronic board that may introduce unmodelled nonlinearities and/or hysteretic effects.

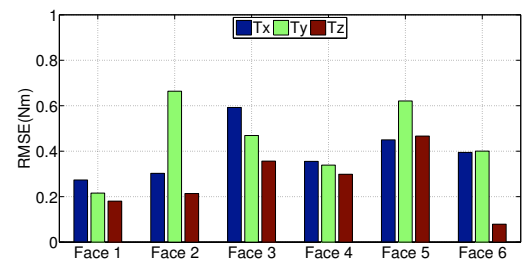

Fig. 10. Loaded Frame Calibration III-A - Torques - RMSE - Only one face at a time is loaded.

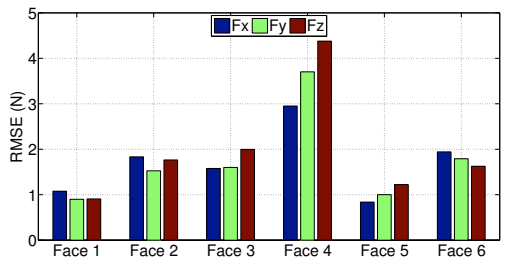

Fig. 11. Loaded Frame Calibration III-A - Forces - RMSE - Two faces simultaneously are loaded (squeeze).

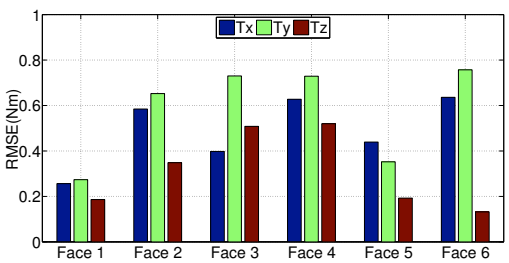

Fig. 12. Loaded Frame Calibration III-A - Torques - RMSE - Two faces simultaneously are loaded (squeeze).

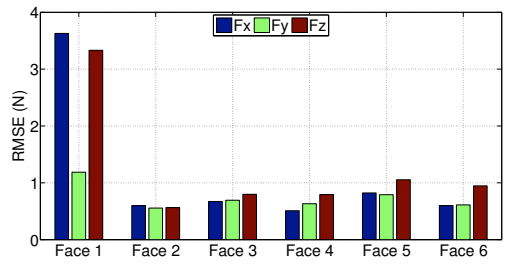

Fig. 13. Unloaded Frame Calibration III-B - Forces - RMSE - Only one face at a time is loaded.

\section{Calibration Matrix Analysis}

In Tab. I the inverse condition number of calibration matrix $(\in[0,1])$ for the Loaded Frame and Unloaded Frame Calibration is reported as an index to describe the mapping isotropy, for different faces connected to the flange. The analysis suggests that, for both calibration methods, no notable anisotropic effect can be associated to a particular face, meaning that the choice of the face during calibration phase is not a critical aspect.

\section{CONTACT POINT RESULTS}

To assess the accuracy of the contact point reconstructions, the algorithm presented in [14] and implemented in [15] was 


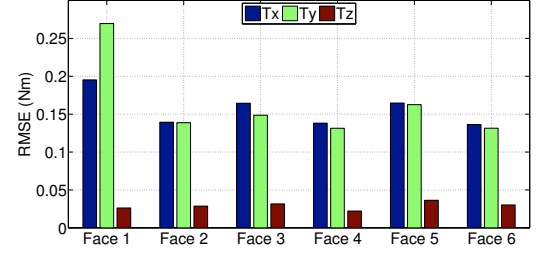

Fig. 14. Unloaded Frame Calibration III-B - Torques - RMSE - Only one face at a time is loaded.

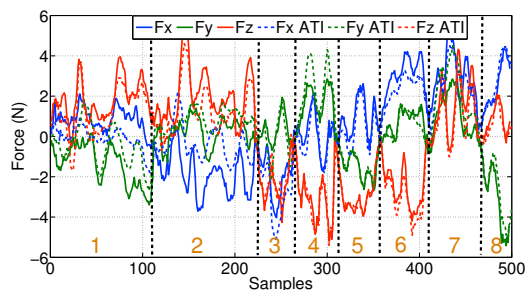

Fig. 15. Unloaded Frame Calibration III-B - Forces - Comparison - Two faces simultaneously are loaded (squeeze).

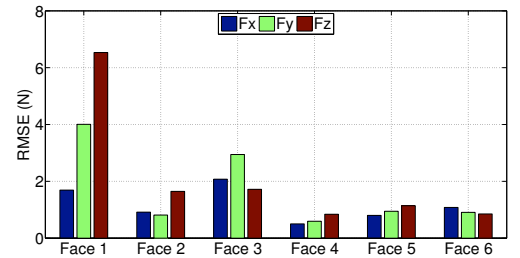

Fig. 16. Unloaded Frame Calibration III-B - Forces - RMSE - Two faces simultaneously are loaded (squeeze).
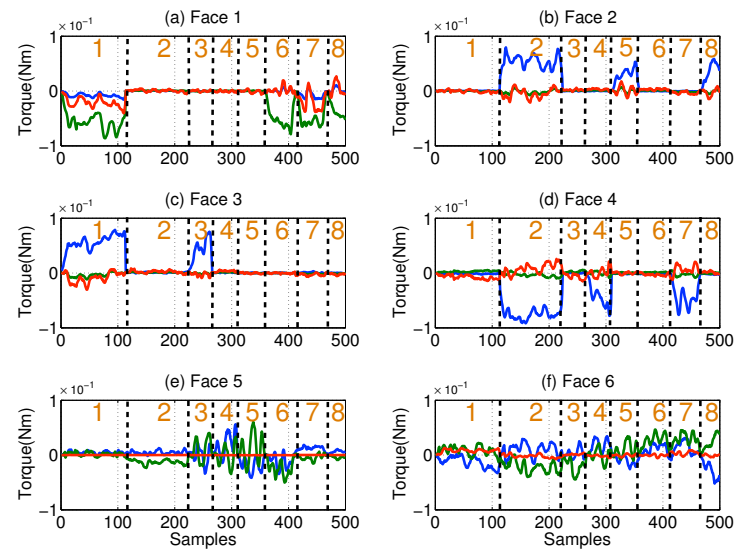

$$
-\mathrm{Tx}-\mathrm{Ty}-\mathrm{Tz}
$$

Fig. 17. Unloaded Frame Calibration III-B - Torques - Identification of loaded faces - Two faces simultaneously are loaded (squeeze).

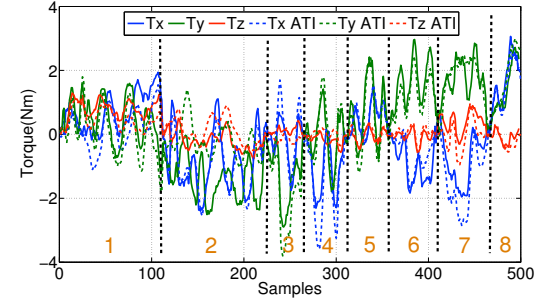

Fig. 18. Unloaded Frame Calibration III-B - Torques - Comparison Two faces simultaneously are loaded (squeeze).

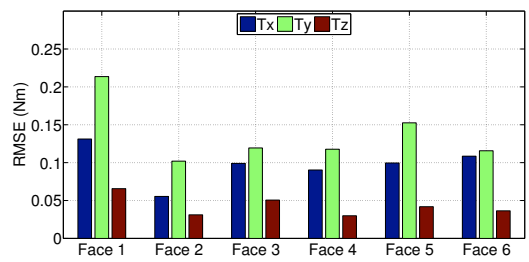

Fig. 19. Unloaded Frame Calibration III-B - Torques - RMSE - Two faces simultaneously are loaded (squeeze).

applied both to the custom made sensor and to the ATI Delta F/T sensor.

The algorithm was employed with a surface patch for the cubical shape (in this case we use only one face of the custom made 36 axis F/T sensor). Then, the same surface patch was fixed on the ATI Delta F/T sensor. The contact surface was a square of $46 \times 46 \mathrm{~mm}$ with 8 points. These points lie on the edges of a $26 \times 26 \mathrm{~mm}$ square.

The experimental task was performed touching the points (one at a time) with a fingertip and then touching the center of the surface patch. As reported in Fig. 21(a) and Fig. 21(b), the accuracy of our sensor after the calibration can be assessed in the order of $3 \mathrm{~mm}$ in the range of practical interest.

\section{EXPERIMENTS}

To put the effective performances of the sensorized object on a test, we performed two experiments. In the first one, we tested the calibration, while in the second one we tracked the contact points during a grasp.

\section{A. Overall Accuracy}

In this experiment we assess the overall correct calibration of the cube. We grasp and touch the custom made sensor

TABLE I

INVERSE CONDITION NUMBER OF DIFFERENT CALIBRATION MATRICES

\begin{tabular}{|c|c|c|}
\hline \hline & \multicolumn{2}{|c|}{ Calibration Methods } \\
\hline Connected Face & Loaded & Unloaded \\
\hline \hline 1 & $1.4 \cdot 10^{-3}$ & $1.1 \cdot 10^{-4}$ \\
\hline 2 & $9.3 \cdot 10^{-4}$ & $9.4 \cdot 10^{-5}$ \\
\hline 3 & $8.3 \cdot 10^{-4}$ & $2.7 \cdot 10^{-4}$ \\
\hline 4 & $9.5 \cdot 10^{-4}$ & $1.9 \cdot 10^{-4}$ \\
\hline 5 & $8.6 \cdot 10^{-4}$ & $2.6 \cdot 10^{-4}$ \\
\hline 6 & $1.2 \cdot 10^{-3}$ & $1.8 \cdot 10^{-4}$ \\
\hline
\end{tabular}


(a) Experiment Snapshot

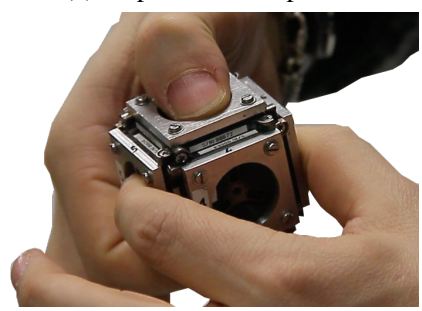

(b) Applied Forces

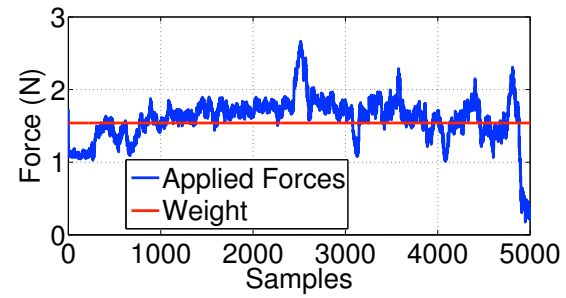

(c) Applied Torques

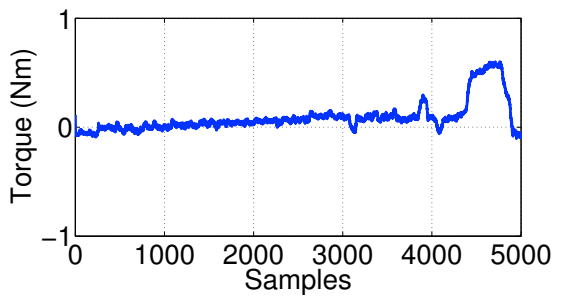

Fig. 20. In fig. (a) we report a snapshot of the experiment for checking the correct behavior of the calibration. In figs. (b) and (c) we report the norm of all the forces an moments applied during the experiment, respectively.

by randomly loading many faces at a time. As performance indicator, we report the norm of the resultant of the contact forces during the experiments.

Indeed, if we consider quasi-static motions of the object, at each time step of the experiment the static force and moment equilibria should hold, that is

$$
\begin{gathered}
\left\|\sum_{i} F_{i}\right\|=\left\|F_{w}\right\|, \\
\left\|\sum_{i} T_{i}\right\|=0
\end{gathered}
$$

where each $F_{i} \in \mathbb{R}^{3} / T_{i} \in \mathbb{R}^{3}$ is the force/torque applied to the $i^{\text {th }}$ face, $F_{w}$ is the object's own weight, and $\|\cdot\|$ is the norm function. The equilibrium of the torques in eq. (14) is considered with respect to the center of gravity of the cube. The calibration used for this experiment is the Unloaded Frame Calibration.

In Fig. 20(a) we show a snapshot of the cube grasp during the experiment performed (for more details see the attached video: PITOExperiments.mp4), while in Figs. 20(b) and 20(c) we report the norm of contact force resultants vs. the object's own weight, and the torque balance during the experiment, respectively. The performances are good and the spikes/noise present in the graph are actually due to the non-static conditions of the test - the acceleration of the object center of gravity is not zero at some instants - due to (a) Marked Patch

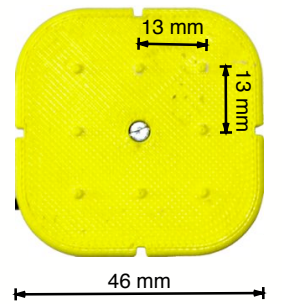

(b) Device Setup

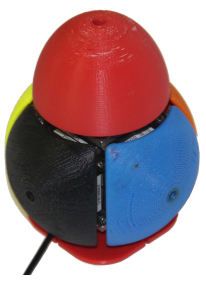

Fig. 21. In fig. (a) we report the patch used for computing the accuracy in contact point reconstruction of the device presented. In fig. (b) we show the sensorized object arrangement for contact detection and reconstruction. (a) Multi DoFs F/T Sensor

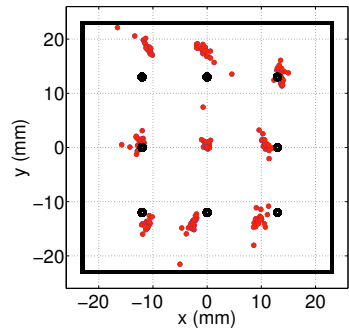

(b) ATI Delta F/T Sensor

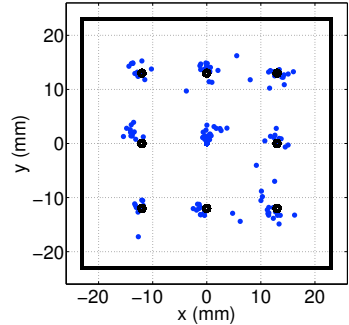

Fig. 22. Contact point algorithm tested with face 5 of the custom made sensor. Reference points (black) were touched with fingertip (one at a time). Red points are the algorithm results (a). Contact point algorithm tested with ATI Delta sensor. Reference points (black) were touched with fingertip (one at a time). Blue points are the algorithm results (b).

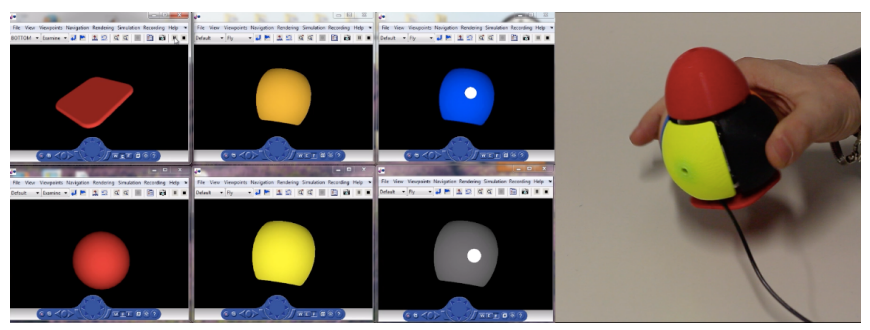

Fig. 23. A snapshot of the contact point detection experiment. On the left side it is possible to notice the contact point detection and reconstruction (white sphere) on a virtual scene while in the left side it is reported the real grasp performed.

impossibility to ensure those conditions when the object is hold in a hand and to the unavoidable hand tremor.

\section{B. Contact Point Detection}

To investigate the correct functionality of the sensorized object we perform two experiments on the contact point detection: in the first one, we determine the accuracy, while in the second one, we check the operating principle of the device.

For both experiments, the algorithm presented in [14] and implemented in [15] was applied and the Unloaded Frame Calibration was used. 
In the first experiment, the algorithm for contact detection on a planar patch was employed (usually employed for cubical shape). In this case, we used only one face of the custom made 36 axis F/T sensor. Then, the same surface patch was fixed on the ATI Delta F/T sensor. The contact surface was a square of $46 \times 46 \mathrm{~mm}$ with 8 points. These points lie on the edges of a $26 \times 26 \mathrm{~mm}$ square.

The experimental task was performed touching the points (one at a time) with the tip of a pencil and then touching the center of the surface patch. As reported in Fig. 21(a) and Fig. 21(b), the accuracy of our sensor after the calibration can be assessed in the order of $3 \mathrm{~mm}$ in the range of practical interest, which is a satisfactory performance for the envisioned use.

In the second experiment, we grasp the sensorized object with the shape arrangement reported in Fig. 21(b). This shape set is composed by four spherical patches (lateral patches of different colors), a semi-ellipsoidal patch (upper red patch), and a planar patch (lower red patch).

During the experiment, we touched all the patches randomly and we compute the contact points on every face. Altough qualitative in nature, the results of this test appear to be appropriate for the expected applications. The entire experiment is documented in the video submission attached to this paper: a snapshot is reported in Fig. 23.

\section{CONCLUSION}

In this paper, we reported on the development of a modular multi-DoF F/T sensor, presented its performances, and gave some examples about its use in the implementation of a sensorized object capable of multi-touch detection. The different calibration methods were described to show how the coupling phenomena inherent to spatial organization of the faces and the lightweight construction of the sensor were tackled. These lead to good performances (tested against an expensive commercial 6-axis sensor) in terms of: (i) ability to detect the touched face, (ii) measured wrench intensity, and (iii) ability to reconstruct the contact centroid position. Thanks to the modular design and the possibility to cover the sensitive faces with surface patches of different geometry, a variety of sensorized objects with different shapes can be realized, as shown in Sec. VI. The peculiar feature that all the components of the contact wrench can be measured on each face with high accuracy, renders it a unique tool in the study of grasp force distribution in humans, with forthcoming use both in neuroscience investigations and robotic applications. Ongoing research is also devoted to the miniaturization of the current faces to obtain small facets. This will allow: (i) to obtain sensorized arbitrarily complex free-form surfaces capable of full wrench estimation per patch and, due to the increased ratio between finger and contact facet characteristic dimensions, (ii) it will mitigate the unavoidable limitation that only a single contact/wrench per patch can be properly detected/measured. While there is much technological work on the way, we believe that the methodology presented in this paper will carry over with small modifications.

\section{ACKNOWLEDGMENT}

This work is supported by the European Research Council under the ERC Advanced Grant no. 291166 SoftHands (A Theory of Soft Synergies for a New Generation of Artificial
Hands), under the CP-IP grant no. 248587 THE Hand Embodied and grant agreement no. 601165 Wearhap (Wearable Haptics for Humans and Robots), within the FP7/2007-2013 program: Cognitive Systems and Robotics and by the grant no. 600918 "PACMAN" - Probabilistic and Compositional Representations of Object for Robotic Manipulation - within the FP7-ICT-2011-9 program "Cognitive Systems".

\section{REFERENCES}

[1] [Online]. Available: http://www.thehandembodied.eu

[2] G. Baud-Bovy and J. F. Soechting, "Two virtual fingers in the control of the tripod grasp," Journal of Neurophysiology, vol. 86, no. 2, pp. 604-615, 2001.

[3] A. Bicchi, M. Gabiccini, and M. Santello, "Modelling natural and artificial hands with sinergie," Phil. Trans. R. Soc. B, vol. 366, pp. 3153-3161, 2011.

[4] M. Santello, M. Flanders, and J. F. Soechting, "Postural hand synergies for tool use," The Journal of Neuroscience, vol. 18, no. 23, pp. 10105 - $10115,1998$.

[5] M. Gabiccini, G. Stillfried, H. Marino, and M. Bianchi, "A datadriven kinematic model of the human hand with soft-tissue artifact compensation mechanism for grasp synergy analysis," in IEEE/RSJ International Conference on Intelligent Robots and Systems, Dec. 2013, pp. 1-8.

[6] R. Triolo, Ed., A Sensorized Glove For Applications in Biomechanics and Motor Control. Cleveland, Ohio: IFESS, 2001.

[7] M. A. Roa, R. Koiva, and C. Castellini, "Experimental evaluation of human grasps using a sensorized object," in Biomedical Robotics and Biomechatronics (BioRob), 2012 4th IEEE RAS \& EMBS International Conference on, 2012, pp. 1662-1668.

[8] Y.-J. Li, B.-Y. Sun, J. Zhang, M. Qian, and Z.-Y. Jia, "Measurement," Measurement, vol. 42, no. 5, pp. 730-736, June 2009.

[9] P. A. Schmidt, E. Maël, and R. P. Würtz, "A sensor for dynamic tactile information with applications in human-robot interaction and object exploration," Robotics and Autonomous Systems, vol. 54, no. 12, pp. 1005-1014, 2006.

[10] F. Beyeler, S. Muntwyler, and B. J. Nelson, "A six-axis mems forcetorque sensor with micro-newton and nano-newtonmeter resolution," Microelectromechanical Systems, Journal of, vol. 18, no. 2, pp. 433441, 2009.

[11] I. Sarakoglou, N. Tsagarakis, and D. Caldwell, "Unita' elettronica di misura per un dispositivo polimorfico per la misura di forze, e dispositivo polimorfico includente la medesima," Patent TO2012A000 890.

[12] — , "Electronic measurement unit for a polymorphous device for force measurement and polymorphous device including the same," Patent WO2 014057479.

[13] A. Serio, M. Gabiccini, I. Sarakoglou, and A. Bicchi, "Calibration and test of a multi-touch tactile object," International Conference of Intelligent Robots and Systems, workshop in Advances in tactile sensing and touch-based human-robot interaction, IROS 2012, Algrave, Portugal, 2012.

[14] A. Bicchi, J. K. Salisbury, and D. L. Brock, "Contact sensing from force measurements," International Journal of Robotics Research, vol. 12, pp. 249-262, 1993.

[15] [Online]. Available: http://www.thehandembodied.eu/tools

[16] R. M. Murray, Z. Li, and S. S. Sastry, "A mathematical introduction to robotic manipulation," 1994.

[17] M. Gabiccini, "A twist exponential approach to gear generation with general spatial motions," MAMT, vol. 44, no. 2, pp. 382-400, Feb. 2009. 